\title{
Ubiquitous Text Interaction
}

Keith Vertanen

Michigan Technological

University

Houghton, MI, USA

vertanen@mtu.edu

\section{Mark Dunlop}

University of Strathclyde

Glasgow, UK

mark.dunlop@cis.strath.ac.uk

\section{Xiaojun Bi}

Stony Brook University

Stony Brook, NY, USA

xjunbi@gmail.com
Kyle Montague

Newcastle University

Newcastle, UK

Kyle.Montague@newcastle.ac.uk

Ahmed Sabbir Arif

Ryerson University

Toronto, Ontario, Canada

asarif@ryerson.ca

\section{Shiri Azenkot}

Cornell Tech

New York, NY, USA

shiri.azenkot@cornell.edu

Permission to make digital or hard copies of part or all of this work for personal or

\begin{abstract}
Computer-based interactions increasingly pervade our everyday environments. Be it on a mobile device, a wearable device, a wall-sized display, or an augmented reality device, interactive systems often rely on the consumption, composition, and manipulation of text. The focus of this workshop is on exploring the problems and opportunities of text interactions that are embedded in our environments, available all the time, and used by people who may be constrained by device, situation, or disability.
\end{abstract}

This workshop welcomes all researchers interested in interactive systems that rely on text input or output. Participants should submit a short position statement outlining their background, past work, future plans, and suggesting a use-case they would like to explore in-depth during the workshop. During the workshop, small teams will form around common or compelling use-cases. Teams will spend time brainstorming, creating low-fidelity prototypes, and discussing their use-case with the group.

Participants may optionally submit a technical paper for presentation as part of the workshop program. The workshop serves to sustain and build the community of text entry researchers who attend $\mathrm{CHI}$. It provides an opportunity for new members to join this community, soliciting feedback from experts in a small and supportive environment. 


\section{Author Keywords}

Text entry; text interaction; mobile interaction.

\section{ACM Classification Keywords}

H.5.2 [Information interfaces and presentation]: Input devices and strategies.

\section{Background}

The consumption and generation of text feature heavily in many of the computer interfaces we use everyday. Ten years ago, computing was primarily performed on desktop computers. Text was displayed on big two-dimensional monitors and user input was primarily via a keyboard and mouse. For most people, desktop computing only constituted a small portion of their day.

Today, mobile devices dominate and computing is as close as the phone in your pocket. Wearable devices such as smartwatches further lower the threshold for initiating computer interactions. Our mobile and wearable devices allow near instant communication with friends and family via text messaging. We can consult our devices for the latest news, to help navigate, or to seek information from the web. Text is still displayed on two-dimensional screens, though often quite small ones. Input is primarily via gestures made on a touch-sensitive screen.

As devices that augment our senses with virtual content move from the research lab to product prototypes (e.g. Google Glass and Microsoft HoloLens), the threshold for interaction will become even lower. In a future where such devices are common, interaction becomes continuous and ever present. The focus of this workshop is on what such a future of always-on interaction embedded into our real-world means for text entry research. Further, we are interested in how to make such a future accessible to all people, regardless of limitations imposed by device, situation, or disability.

The term "text entry" is perhaps too narrow. While the fluid and efficient input of text is certainly a challenge, it is only one part of the problem. Interfaces also need to provide users with powerful, fluid, and task-appropriate text interaction. Prominent amongst these are supporting editing and correcting [1, 9, 25, 18], formatting of text [7], personalization [8], and context-awareness [10,11].

The design of text interactions also requires special attention to the needs and capabilities of diverse types of users. For example children [21, 2], older adults [27, 12], users with motor disabilities [26], and users with visual impairments [3, 23, 17, 19, 22]. Further, interacting while in augmented or virtual reality poses many design challenges $[4,15,16,20]$.

This workshop has the following goals:

- Identify the research challenges associated with enabling rich text interaction embedded and intelligently integrated into the world around us.

- Identify the research challenges caused by input or output modalities being constrained by device, situation, or disability.

- Explore and discuss early work that addresses these challenges.

- Connect researchers with allied interests and complementary talents to facilitate progress in addressing these challenges.

\section{Organizers}

The organizers are all widely published experts on text entry. They have all published extensively in the $\mathrm{HCl}$ text entry 
community and often collaborate with researchers from a variety of communities, such as Augmentative and Alternative Communication (AAC), Natural Language Processing (NLP), and speech recognition.

Keith Vertanen is an Assistant Professor at Michigan Technological University. He specializes in designing intelligent interactive systems that leverage uncertain input technologies. This includes input via speech, touchscreens, and eye-gaze. A particular focus of his research is on systems that enhance the capabilities of users with permanent or situationally-induced disabilities. Dr. Vertanen is the main contact person for this workshop.

Kyle Montague is a Research Associate at Open Lab, Newcastle University. His research focuses on digital technologies and accessibility; in particular solutions to support non-visual text-entry and interface adaptation.

Mark Dunlop is a Senior Lecturer at the University of Strathclyde. He has been publishing in mobile text entry since 1999 and is currently running a research council project on text entry for older adults. His interests include both the underlying algorithms for smarter predictive text entry and novel solutions for user input on varying devices. As well as academic research, he has conducted usability studies on mobile text entry for commercial developers.

Xiaojun Bi is an Assistant Professor at Stony Brook University. Prior to joining Stony Brook, he was a Research Scientist at Google. Xiaojun Bi's research focuses on building interactive systems, designing interaction techniques, and studying fundamental issues of user interface design especially on mobile devices. He has pioneered a number of techniques for mobile text entry systems, pen and touch based interactive systems, and large display interaction. His innovations such as keyboard correction and completion algorithm, bimanual gesture typing, and personalizing language models for text entry have been integrated into the Android keyboard used by more than 100 million users.

Ahmed Sabbir Arif is a Postdoctoral Research Fellow at Ryerson University. As a researcher, his goal is to make computer technologies accessible to everyone by developing natural and intuitive input and interaction techniques. A major thread of his work focuses on smarter solutions for text entry, error correction, and editing on various devices. His other interests include text entry evaluations, and multimodal, predictive, and international text entry techniques.

Shiri Azenkot is an Assistant Professor at the Jacobs Technion-Cornell Institute at Cornell Tech, Cornell University. Her research interests are in accessibility, focusing on people with visual and reading disabilities, and interaction techniques on new platforms.

\section{Website}

Our workshop website is located at http://www.textentry.org/ chi2017. The site includes the call for participation, a copy of this workshop proposal paper, and links to the organizers. Details of the one-day workshop program will be posted once details are finalized. The website also provides details about how to subscribe to the text entry research mailing list that was established as part of last year's workshop.

\section{Pre-Workshop Plans}

We have written a Call for Participation that we will send to a variety of mailing lists including: chi-Announcements, access-announcements, SIG-SLPAT (Speech and Language Processing for Assistive Technologies), and our text entry research mailing list. We will also contact authors of position papers from the text entry workshops that occurred at CHI'12 [14], CHI'13 [13], CHl'15 [5], and CHl'16 [24]. 
The organizers will reach out to personal contacts in an effort to solicit broad participation. We will identify researchers outside $\mathrm{HCl}$ who may be interested in participating and encourage them to submit a position statement. Based on feedback from last year's workshop, we plan to make explicit efforts this year to reach out to industrial researchers.

Our past workshop participants enjoyed the discussion and community aspects of the workshop the most. As such, we believe having a "lightweight" position statement as the requirement for attendance will improve our already healthy participant numbers. It will also allow us to make acceptance decisions in a timely manner. Thus this year we require participants submit just a short position statement outlining their background, past work, future plans, and suggesting a use-case they would like to explore in-depth during the workshop. Statements will be reviewed by the workshop organizers to ensure participants have a background or interest in the workshop's theme. We anticipate being able to invite most interested participants.

Some participants may additionally want to present a technical paper. Participants selected on the basis of their position statement may optionally submit a longer, more technical paper for possible inclusion in the workshop program. The deadline for this optional submission will be in late March, allowing authors more time to prepare their submission. Participants will receive at least two full reviews. Past participants, in particular early career researchers, have used the written reviews and feedback at the workshop to improve and extend their work for later successful publication at $\mathrm{CHI}$ and other venues.

Prior to the workshop, the organizers will create a list of the use-cases suggested by attendees, grouping them by similarity and popularity. This list will help organize small groups in the second portion of the workshop.

\section{Workshop Structure}

This is a full one-day workshop. We plan to have two morning paper sessions. Participants of accepted technical papers will give 10-minute talks followed by a 10-minute period for questions and discussion. After the paper sessions, participants will be invited to continue the discussion at a group lunch.

After lunch, we will have a one-hour Show-and-Tell session. This was a new and popular feature added to last year's workshop. This year, we will encourage all participants to demo systems regardless of whether it is related to a paper submission or not. As some participants may not have work of the nature or maturity to be demonstrated, the Showand-Tell session will also have poster presentations.

The next hour session will consist of exploring and discussing future-looking use-cases as suggested by participants in their position statements. Small groups will form around a particular use scenario. Each group will have an hour to discuss the scenario, proposing possible interactive systems, perhaps developing low-fidelity prototypes of their ideas. Groups will identify the research challenges and open questions germane to their scenario.

In the final hour session, each team will present a summary of what they discussed including a walk through of their proposed best idea for an interactive system addressing the scenario. This will be followed by a group discussion about the scenario and the proposed system.

At the end of the last session, we will have an open discussion about what participants thought about the workshop and its format. We will also solicit ideas for themes for future workshops and other community events. Towards our community-building goal, workshop participants will be encouraged to join the text entry research mailing list that was 
established as part of last year's workshop.

\section{Post-Workshop Plans}

We expect a number of important outcomes from this workshop. First, this workshop continues our effort to build and sustain a community of text entry researchers at $\mathrm{CHI}$. Our workshop builds upon the enthusiasm and feedback received from past participants at the successful text entry workshops held at CHI'12 [14], CHI'13 [13], CHI'15 [5], CHI'16 [24], and the CHI'14 text entry SIG [6]. To help participants get to know each other before the workshop, and to connect with each other afterward, we will share a folder with participants' position statements with all attendees.

Second, we hope the workshop, and in particular the group use-case exploration portion, will yield a variety of futurelooking visions of text interaction in a post-desktop and post-mobile world. Participants who share an interest in a particular use-case explored at the workshop will be encouraged to pursue further collaborative work after the workshop. Such collaborations have the potential to bring together researchers with complementary expertise to create and evaluate new interactive techniques that would be difficult for any one person to pursue on their own.

Third, authors of accepted workshop papers will be encouraged to place their papers on the workshop's website. Additionally, we will encourage participants with demos to submit short videos for inclusion on the website.

\section{Call for Participation}

We invite position statements for the $\mathrm{CHI} 2017$ Workshop on Ubiquitous Text Interaction. This one-day workshop offers an interdisciplinary forum for both practitioners and academics interested in interactive systems that rely on text input or output. A particular focus this year is on exploring the problems and opportunities of text interactions that are embedded in our environments, available at all times, and used by people who may be constrained by device, situation, or disability. For example, text interfaces using wearable devices, head-mounted displays, or interaction when visual feedback is limited or non-existent.

\section{Early submission deadline: December 20, 2016 \\ Normal submission deadline: February 21, 2017}

Interested participants should submit a short position statement outlining their background, past work, future plans, and suggesting a use-case they would like to explore during the workshop. During the workshop, small teams will form around compelling use-cases. Teams will spend time brainstorming, creating low-fidelity prototypes, and then presenting and discussing their use-case with the group.

Participants will be selected on the basis of the quality of their position statement. All workshop participants must register for both the workshop and for at least one day of the $\mathrm{CHI} 2017$ conference.

In addition to a position statement, participants are encouraged to submit an optional technical paper for possible inclusion in the workshop program. The separate deadline for paper submission is March 31, 2017. Papers should be in $\mathrm{CHI}$ Extended Abstracts Format (maximum eight pages excluding references). Papers will be peer-reviewed with accepted papers being presented as part of the workshop program as a talk, poster, or demo. At least one author must register and attend the workshop.

For further information, see our workshop website at http: //www.textentry.org/chi2017. 
How to submit:

- Write a position statement in the CHI Extended Abstracts Format (maximum four pages excluding references).

- Each statement should include a biography, and a summary of any past work relevant to the workshop theme.

- Your statement should identify a future-looking text interaction use-case you would like to explore in-depth during the workshop.

- Email your position statement in PDF format to textinteraction@gmail.com

Important dates:

- Early submission deadline: December 20, 2016.

- Early acceptance notification: December 23, 2016.

- Normal submission deadline: February 21, 2017.

- Normal acceptance notification: February 24, 2017.

- Workshop: Saturday May 6, 2017, Denver, Colorado.

Organizers:

- Keith Vertanen, Michigan Technological University, USA.

- Kyle Montague, Newcastle University, UK.

- Mark Dunlop, University of Strathclyde, UK

- Xiaojun Bi, Stony Brook University, USA.

- Ahmed Sabbir Arif, Ryerson University, Canada.

- Shiri Azenkot, Cornell Tech, USA.

\section{References}

[1] Ahmed Sabbir Arif, Sunjun Kim, Wolfgang Stuerzlinger, Geehyuk Lee, and Ali Mazalek. 2016a. Evaluation of a Smart-Restorable Backspace Technique to Facilitate Text Entry Error Correction. In Proceedings of the $2016 \mathrm{CHI}$ Conference on Human Factors in Computing Systems (CHI '16). ACM, New York, NY, USA, 5151-5162. DOI : http://dx.doi.org/10.1145/ 2858036.2858407
[2] Ahmed Sabbir Arif, Cristina Sylla, and Ali Mazalek. 2016b. Learning New Words and Spelling with Autocorrections. In Proceedings of the 2016 ACM on Interactive Surfaces and Spaces (ISS '16). ACM, New York, NY, USA, 409-414. DOI : http://dx.doi.org/10.1145/ 2992154.2996790

[3] Shiri Azenkot, Jacob O. Wobbrock, Sanjana Prasain, and Richard E. Ladner. 2012. Input Finger Detection for Nonvisual Touch Screen Text Entry in Perkinput. In Proceedings of Graphics Interface 2012 (GI '12). Canadian Information Processing Society, Toronto, Ontario, Canada, 121-129. http://dl.acm.org/citation. cfm?id=2305276.2305297

[4] Doug A Bowman, Christopher J Rhoton, and Marcio S Pinho. 2002. Text input techniques for immersive virtual environments: An empirical comparison. In Proceedings of the Human Factors and Ergonomics Society Annual Meeting, Vol. 46. SAGE Publications, 2154-2158.

[5] James Clawson, Ahmed Sabbir Arif, Stephen Brewster, Mark Dunlop, Per Ola Kristensson, and Antti Oulasvirta. 2015. Text Entry on the Edge. In Proceedings of the 33rd Annual ACM Conference Extended Abstracts on Human Factors in Computing Systems (CHI EA '15). ACM, New York, NY, USA, 2381-2384. DOI : http://dx.doi.org/10.1145/2702613.2702660

[6] James Clawson, Stephen A. Brewster, Mark Dunlop, Per Ola Kristensson, Poika M. Isokoski, Antti Oulasvirta, Keith Vertanen, and Annalu Waller. 2014. The Usability of Text Entry Systems Now and in the Future. In $\mathrm{CHI}$ '14 Extended Abstracts on Human Factors in Computing Systems (CHI EA '14). ACM, New York, NY, USA, 1139-1142. DOI : http://dx.doi.org/10.1145/2559206.2559217

[7] David Dearman, Amy Karlson, Brian Meyers, and Ben Bederson. 2010. Multi-modal Text Entry and Selection 
on a Mobile Device. In Proceedings of Graphics Interface 2010 (Gl '10). Canadian Information Processing Society, Toronto, Ont., Canada, Canada, 19-26. http://dl.acm.org/citation.cfm?id=1839214.1839219

[8] Andrew Fowler, Kurt Partridge, Ciprian Chelba, Xiaojun Bi, Tom Ouyang, and Shumin Zhai. 2015. Effects of Language Modeling and Its Personalization on Touchscreen Typing Performance. In Proceedings of the 33rd Annual ACM Conference on Human Factors in Computing Systems (CHI '15). ACM, New York, NY, USA, 649-658. DOI : http://dx.doi.org/10.1145/2702123. 2702503

[9] Vittorio Fuccella, Poika Isokoski, and Benoit Martin. 2013. Gestures and Widgets: Performance in Text Editing on Multi-touch Capable Mobile Devices. In Proceedings of the SIGCHI Conference on Human Factors in Computing Systems $(\mathrm{CH}$ '13). ACM, New York, NY, USA, 2785-2794. DOI : http://dx.doi.org/10.1145/2470654.2481385

[10] Mayank Goel, Leah Findlater, and Jacob O. Wobbrock. 2012. WalkType: using accelerometer data to accomodate situational impairments in mobile touch screen text entry. In $\mathrm{CHI}$ Conference on $\mathrm{Hu}$ man Factors in Computing Systems, CHI '12, Austin TX, USA - May 05 - 10, 2012. 2687-2696. DOI : http://dx.doi.org/10.1145/2207676.2208662

[11] Maryam Kamvar and Shumeet Baluja. 2007. The Role of Context in Query Input: Using Contextual Signals to Complete Queries on Mobile Devices. In Proceedings of the 9th International Conference on Human Computer Interaction with Mobile Devices and Services (MobileHCl '07). ACM, New York, NY, USA, 405-412. DOI : http://dx.doi.org/10.1145/1377999.1378046

[12] Andreas Komninos, Emma Nicol, and Mark D. Dunlop. 2015. Designed with Older Adults to Support Better Error Correction in SmartPhone Text Entry: The
MaxieKeyboard. In Proceedings of the 17th International Conference on Human-Computer Interaction with Mobile Devices and Services Adjunct (MobileHCl '15). ACM, New York, NY, USA, 797-802. DOI : http://dx.doi.org/10.1145/2786567.2793703

[13] Per Ola Kristensson, Stephen Brewster, James Clawson, Mark Dunlop, Leah Findlater, Poika Isokoski, Benoît Martin, Antti Oulasvirta, Keith Vertanen, and Annalu Waller. 2013. Grand Challenges in Text Entry. In CHI '13 Extended Abstracts on Human Factors in Computing Systems (CHI EA '13). ACM, New York NY, USA, 3315-3318. DOI : http://dx.doi.org/10.1145/ 2468356.2479675

[14] Per Ola Kristensson, James Clawson, Mark Dunlop, Poika Isokoski, Brian Roark, Keith Vertanen, Annalu Waller, and Jacob Wobbrock. 2012. Designing and Evaluating Text Entry Methods. In CHI '12 Extended Abstracts on Human Factors in Computing Systems (CHI EA '12). ACM, New York, NY, USA, 2747-2750. DOI : http://dx.doi.org/10.1145/2212776.2212711

[15] Minkyung Lee and Woontack Woo. 2003. ARKB: 3D vision-based Augmented Reality Keyboard. In Proceeding of the 13th International Conference on Artificial Reality and Telexistence.

[16] Mark McGill, Daniel Boland, Roderick Murray-Smith, and Stephen Brewster. 2015. A Dose of Reality: Overcoming Usability Challenges in VR Head-Mounted Displays. In Proceedings of the 33rd Annual ACM Conference on Human Factors in Computing Systems (CHI '15). ACM, New York, NY, USA, 2143-2152. DOI : http://dx.doi.org/10.1145/2702123.2702382

[17] Hugo Nicolau, João Guerreiro, Tiago Guerreiro, and Luís Carriço. 2013. UbiBraille: Designing and Evaluating a Vibrotactile Braille-reading Device. In Proceedings of the 15th International ACM SIGACCESS Conference on Computers and Accessibility (ASSETS 
'13). ACM, New York, NY, USA, Article 23, 8 pages. DOI : http://dx.doi.org/10.1145/2513383.2513437

[18] Hugo Nicolau, Kyle Montague, Tiago Guerreiro, João Guerreiro, and Vicki L. Hanson. 2014. B\#: Chord-based Correction for Multitouch Braille Input. In Proceedings of the 32Nd Annual ACM Conference on Human Factors in Computing Systems (CHI 14). ACM, New York, NY, USA, 1705-1708. DOI : http://dx.doi.org/10.1145/2556288.2557269

[19] Hugo Nicolau, Kyle Montague, Tiago Guerreiro, André Rodrigues, and Vicki L. Hanson. 2015. Typing Performance of Blind Users: An Analysis of Touch Behaviors, Learning Effect, and In-Situ Usage. In Proceedings of the 17th International ACM SIGACCESS Conference on Computers \&\#38; Accessibility (ASSETS '15). ACM, New York, NY, USA, 273-280. DOI : http://dx.doi.org/10.1145/2700648.2809861

[20] Sebastian Pick, Andrew S. Puika, and Torsten W. Kuhlen. 2016. SWIFTER: Design and evaluation of a speech-based text input metaphor for immersive virtual environments. In 2016 IEEE Symposium on 3D User Interfaces, 3DUI 2016, Greenville, SC, USA, March 19-20, 2016. 109-112. DOI : http://dx.doi.org/10.1109/3DUI.2016.7460039

[21] Janet C. Read, Stuart MacFarlane, and Chris Casey. 2002. Oops! Silly Me! Errors in a Handwriting Recognition-based Text Entry Interface for Children. In Proceedings of the Second Nordic Conference on Human-computer Interaction (NordiCHI '02). ACM, New York, NY, USA, 35-40. DOI : http://dx.doi.org/10. 1145/572020.572026

[22] André Rodrigues, Hugo Nicolau, Kyle Montague, Luís Carriço, and Tiago Guerreiro. 2016. Effect of Target Size on Non-visual Text-entry. In Proceedings of the 18th International Conference on Human-Computer Interaction with Mobile Devices and Services (Mo-
bileHCl '16). ACM, New York, NY, USA, 47-52. DOI : http://dx.doi.org/10.1145/2935334.2935376

[23] Caleb Southern, James Clawson, Brian Frey, Gregory Abowd, and Mario Romero. 2012. An Evaluation of BrailleTouch: Mobile Touchscreen Text Entry for the Visually Impaired. In Proceedings of the 14th International Conference on Human-computer Interaction with Mobile Devices and Services (MobileHCl '12). ACM, New York, NY, USA, 317-326. DOI : http://dx.doi.org/10.1145/2371574.2371623

[24] Keith Vertanen, Mark Dunlop, James Clawson, Per Ola Kristensson, and Ahmed Sabbir Arif. 2016. Inviscid Text Entry and Beyond. In Proceedings of the 2016 CHI Conference Extended Abstracts on Human Factors in Computing Systems (CHI EA '16). ACM, New York, NY, USA, 3469-3476. DOI : http: //dx.doi.org/10.1145/2851581.2856472

[25] Keith Vertanen and Per Ola Kristensson. 2009. Parakeet: A Continuous Speech Recognition System for Mobile Touch-screen Devices. In Proceedings of the 14th International Conference on Intelligent User Interfaces (IUI '09). ACM, New York, NY, USA, 237-246. DOI : http://dx.doi.org/10.1145/1502650.1502685

[26] Jacob O. Wobbrock, Brad A. Myers, and John A. Kembel. 2003. EdgeWrite: A Stylus-based Text Entry Method Designed for High Accuracy and Stability of Motion. In Proceedings of the 16th Annual ACM Symposium on User Interface Software and Technology (UIST '03). ACM, New York, NY, USA, 61-70. DOI: http://dx.doi.org/10.1145/964696.964703

[27] Patricia Wright, Christine Bartram, Nick Rogers, Hazel Emslie, Jonathan Evans, Barbara Wilson, and Steve Belt. 2000. Text entry on handheld computers by older users. Ergonomics 43, 6 (2000), 702-716. 